\title{
Anti-Basement Membrane Glomerulonephritis
}

National Cancer Institute

\section{Source}

National Cancer Institute. Anti-Basement Membrane Glomerulonephritis. NCI Thesaurus. Code C35798.

Inflammation of the glomeruli secondary to presence of autoantibodies directed at specific antigenic targets within the glomerular basement membrane, causing hematuria, proteinuria, and impaired renal function. 